\title{
Personalized accessible wayfinding for people with disabilities through standards and open geospatial platforms in smart cities
}

\author{
Bradley Wheeler ${ }^{1}$, Meirman Syzdykbayev ${ }^{1 *}$ (D, Hassan A. Karimi ${ }^{1}$, Raanan Gurewitsch ${ }^{2}$ and Yanbo Wang ${ }^{2}$
}

\begin{abstract}
Of the many features that smart cities offer, safe and comfortable mobility of pedestrians within the built environment is of particular importance. Safe and comfortable mobility requires that the built environments of smart cities be accessible to all pedestrians, mobility abled and mobility impaired, given their various mobility needs and preferences. This, coupled with advanced technologies such as wayfinding applications, pedestrians can get assistance in finding the best pathways at different locations and times. Wayfinding applications comprise two components, a database component containing accessibility data, and appropriate algorithms that can utilize accessibility data to meet the mobility needs and preferences of all individuals. While wayfinding applications that provide accessibility on both permanent (e.g., steps) and temporary (e.g., snow) pathways are becoming available, there is a gap in current solutions. There are two elements in the gap, one is that the accessibility data used for finding accessible pathways for people with disabilities are not compliant to the widely agreed upon and available standards, another is that the accessibility data are not available in free and open platforms so that they can be used by developers to develop personalized wayfinding applications and services. To fill this gap, in this paper, we propose a new extension in CityGML with accessibility data. We demonstrate the benefits of the new extension by testing various route options within a city. These route options clearly show the differences between commonly (shortest and fastest) requested and produced pathways and accessible pathways that are feasible and preferred by people who are mobility impaired, such as wheelchair users.
\end{abstract}

Keywords: Wayfinding, Accessible pathways, CityGML, Americans with disabilities act accessibility guidelines (ADAAG)

\section{Introduction}

One of the main goals of smart cities is to make pedestrian travels safe and comfortable. This requires the interactions between two activities, one relates to the built environment and another to technologies that are used to assist people to utilize smart city features. The built environment must contain accessible pathways that

\footnotetext{
* Correspondence: mis180@pitt.edu

${ }^{1}$ Geoinformatics Laboratory, School of Computing and Information, University of Pittsburgh, 135 N Bellefield Ave, Pittsburgh, PA 15213, USA Full list of author information is available at the end of the article
}

allow movement of all pedestrians, mobility abled and mobility impaired, between different locations and with ease (note, we refer to "pathways" in this paper as routes between origins and destinations which are designated for walking by pedestrians, and refer to "accessible pathways" as pedestrian routes that can be taken by all individuals, mobility abled or mobility impaired). Building accessible pathways for all pedestrians is the responsibility of the city planners and engineers whose main objective related to mobility is to enhance the mobility experiences of all pedestrians. On the other hand, the 
technologies (devices and tools) considered for smart cities must facilitate all pedestrians' travel within the built environment by finding accessible pathways that meet their special needs and preferences. Mobility technologies, in particular wayfinding applications, are usually developed by technologists with the aim of providing personalized route solutions.

Wayfinding applications consist of two core components, a mobility database and a routing algorithm. The mobility database contains data on all transportation pathways (roadways and other places) in outdoor environments and the routing algorithm utilizes the data in the database to find desired routes. While wayfinding applications have much advanced, they lack

a) mobility databases with sufficient mobility data for pedestrian walking on sidewalk segments [1];

b) appropriate standards-based accessibility data for accessible wayfinding of people with disabilities (PWDs); and

c) appropriate routing algorithms for finding pathways suitable for PWDs.

Furthermore, besides the appropriateness of data content (general mobility and accessibility data), the database must be available for free and in open geospatial platforms to the public and all stakeholders, including city planners, transportation engineers, technologists, and residents, among others. To better understand the gap in current wayfinding applications, Figure 1 shows two example walking routes produced by Google Map. As it is shown in this figure, while a walking route between a pair of locations is requested, Google Maps produces a route that requires the pedestrian walk on roads, at least for parts of the travel, instead of all on sidewalks. Whether or not all walking is on sidewalk is important in that while the produced route may be usable by people with no mobility challenges, it is not feasible for people who use wheelchairs or have other mobility challenges. This is because wheelchair users avoid traveling on roads due to safety and there is no information about accessibility (e.g., barriers) on the produced route.

Wayfinding applications for smart cities must: contain sufficient sidewalk data for pedestrians; contain appropriate accessibility information on sidewalk segments for PWDs; be widely available to all stakeholders (city planners, software developers, etc.); and support routing algorithms that utilize accessibility data in conjunction with other data to find personalized accessible pathways. Since current wayfinding applications do not support all these requirements fully or adequately, in this paper, we fill this gap by developing a new extension and include it in CityGML [2] in order to allow the development of wayfinding applications that feature accessible pathways for mobility challenged individuals. To this end, our proposed extension is focused on sidewalk data, addressing the mobility of the general pedestrian needs and preferences, and in particular includes accessibility data standards delineated from the Americans with Disabilities Act Accessibility Guidelines (ADAAG) [3], addressing the needs and preferences of PWDs. Our choice of the ADAAG standards stems from the observations that they contain the appropriate accessibility information, which can be used as a qualifier for various sidewalk parameters such as width, slope, etc., for people with different disabilities and have been well established and widely used since 1990. Our choice of the CityGML platform stems from the observations that it is free and open, it features standards by different communities, including the World Wide Web Consortium and the Open Geospatial Consortium, it is widely used by researchers and practitioners for a variety of city planning activities, it is considered by some stakeholders as the platform of choice for smart cities, and it already includes a data model suitable for pedestrians. Our proposed application domain extension (ADE) in CityGML is unique in that by utilizing the ADAAG it addresses the mobility needs and preferences of all individuals including those who are mobility impaired. We call this new extension the ADE for accessible pathways(ADE-AP). Figure 2 shows some of the main data in ADE-AP which include 3D data on city objects, such as roads, buildings, vegetation, and road signs. Wayfinding applications can utilize the data in our proposed ADE-AP to provide accessible pathways that meet different needs and preferences by all pedestrians, mobility abled and mobility imapired.

The main contribution of the paper is ADE-AP, a new extension in CityGML, to include and account for all sidewalk conditions needed for wayfinding solutions of all pedestrians, mobility abled and mobility impaired. We demonstrate the benefits of ADE-AP by comparing accessible pathways for wheelchair users with pedestrian pathways provided by popular and widely used services like Google Maps.

The rest of the paper is organized as follows. The background section provides background information on pedestrian wayfinding, ADAAG, wayfinding for PWDs, wayfinding applications, and ontology for wayfinding of PWDs. CityGML and ADE-AP provides an overview of CityGML and describes our proposed ADE-AP, how it fits in CityGML, and how it can be used. Experiments describes our experiments to generate ADE-AP -driven accessible pathways and compare them with pedestrian pathways provided by other services. Results and discussion discusses the result of the comparison using specific metrics. We 


\section{a}

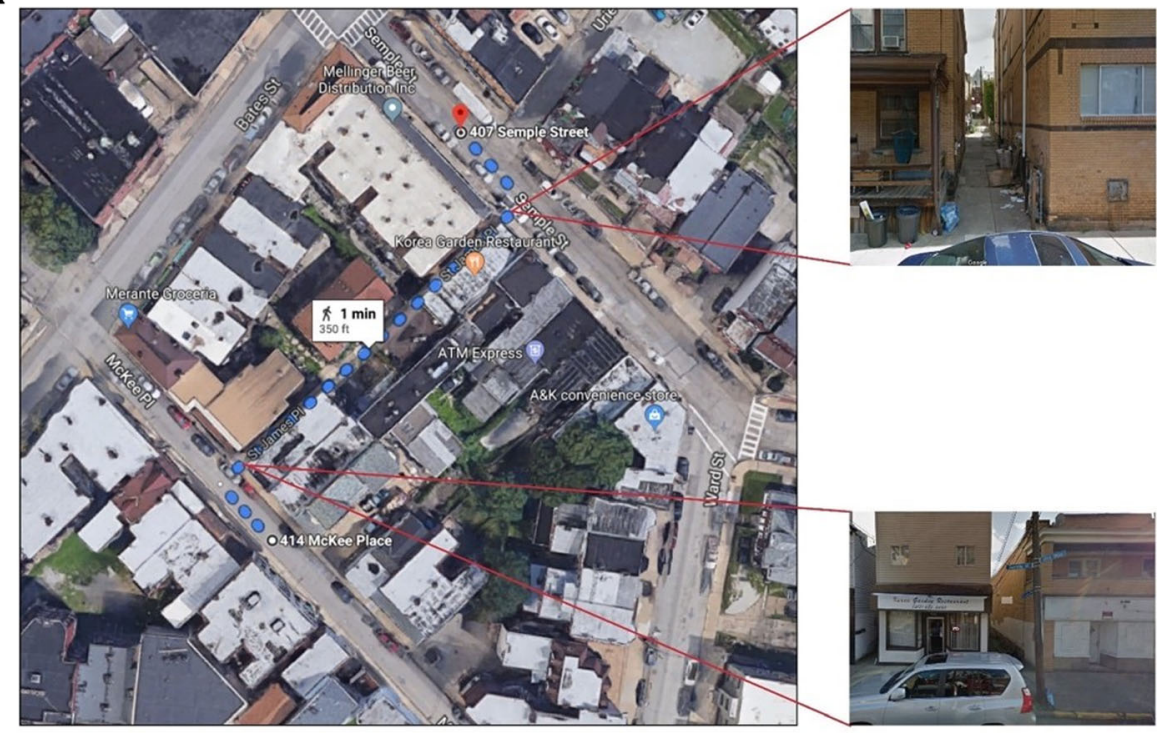

\section{b}

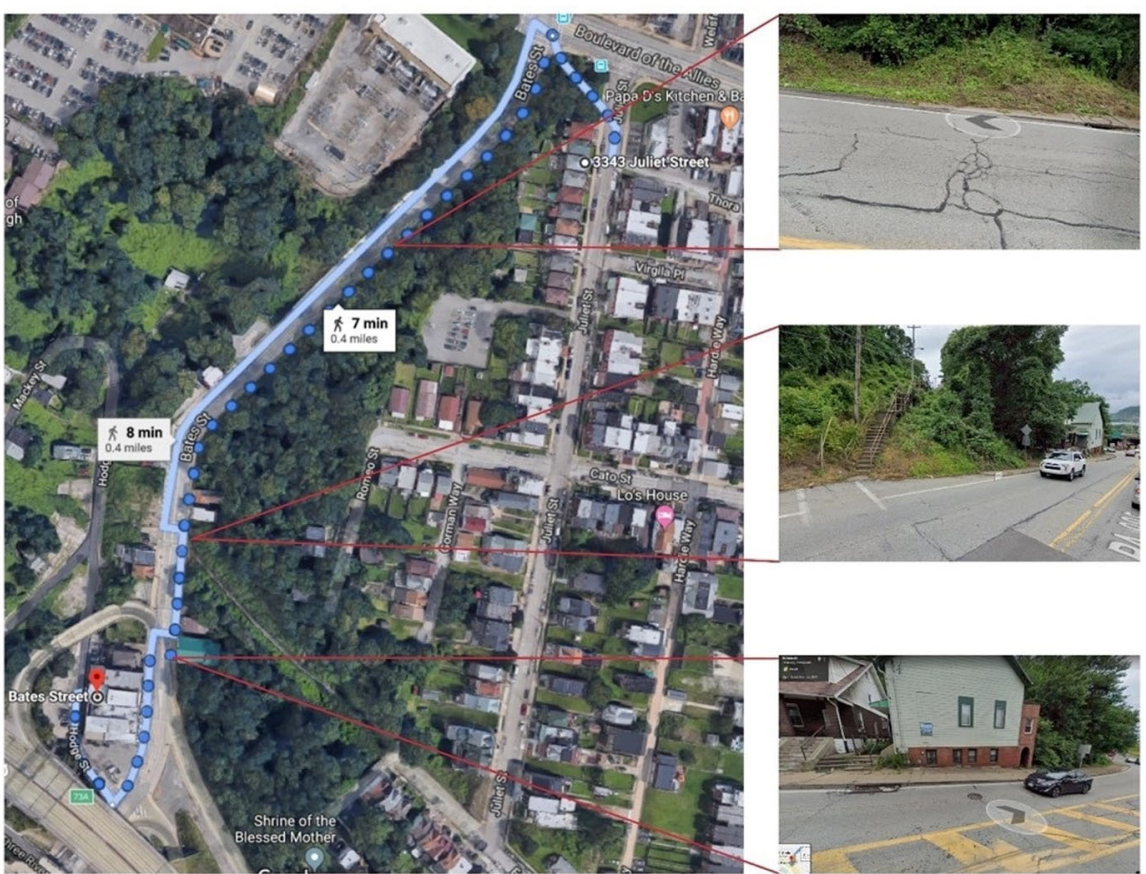

Fig. 1 Pedestrian pathways provided by Google Maps

finish with our conclusion and future research directions.

\section{Background}

Since personalized accessible wayfinding applications require an understanding of some different key current and emerging approaches, techniques, and technologies, in this section we overview pedestrian wayfinding, ADAAG, wayfinding for PWDs, wayfinding applications, and ontology for wayfinding of PWDs.

\section{Pedestrian wayfinding}

Pedestrian wayfinding is concerned with optimizing travel for humans on foot. This is distinct from vehicle wayfinding for the reason that the parameters involved in pedestrian wayfinding do not overlap those in vehicle wayfinding. Pedestrian wayfinding includes additional physical, physiological safety, and mental satisfaction [1] parameters that are not present in vehicle wayfinding. Steps or steep inclines may impact physical conditioning which are not consideration in vehicle wayfinding. Pathways width or 


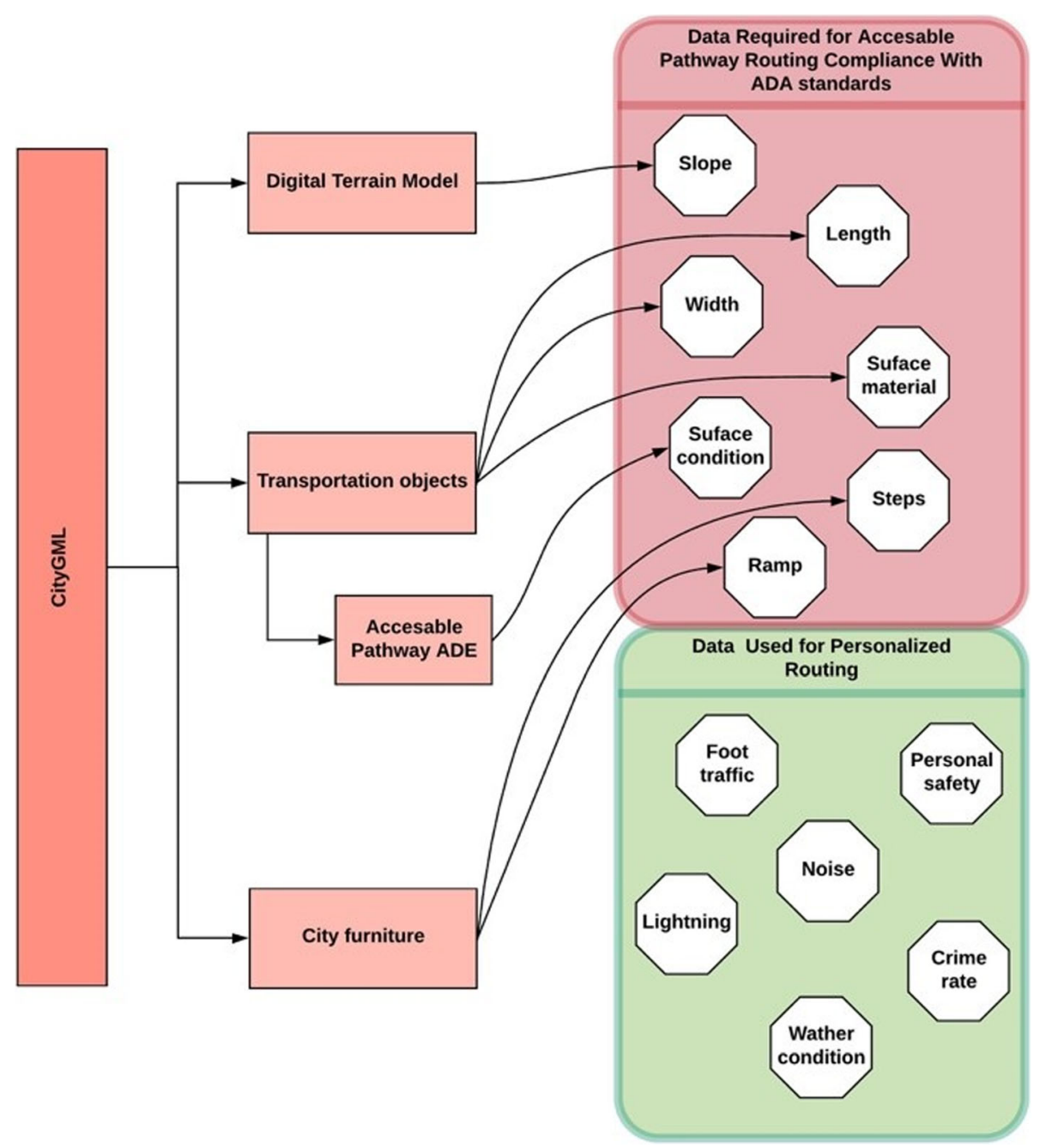

Fig. 2 Our proposed ADE-AP for CityGML impaired

proximity to busy roads may impact the physiological safety felt by a pedestrian and the mental satisfaction received while navigating a given route. This does not leave pedestrian wayfinding without any advantages over vehicle wayfinding. Pedestrians can take paths that traverse squares, parks, grasslands, or pedestrian malls [4]. Pedestrians can also utilize paths that go through buildings which prove advantageous in areas such as large campuses or hospitals. In addition to the distinct needs and preferences pedestrians have over vehicles in wayfinding, PWDs also have distinct requirements for wayfinding that need special attention. This is to say that while it is important to emphasize the distinct needs for pedestrian wayfinding and how they differ from the requirements for vehicle wayfinding, the wayfinding needs and preferences of PWDs only partially overlap with those of the general population.

Of equal importance is how people find their way while in vehicles compared to when they are on foot. While instructions for turning at intersections is ideal for vehicle travel, research has shown this is not the optimal mode of instruction for pedestrian wayfinding $[4,5]$. Landmarks have been shown to be the most useful cue for pedestrian navigation and are absent in most commercial wayfinding solutions. Instructions such as "turn left at the church" have been proven more useful in pedestrian navigation, particularly as pedestrians travel much slower than vehicles and can take in more information in each space. Landmarks have been shown to make instructions for navigation significantly more manageable for pedestrian wayfinding [6]. Landmarks, such as buildings, have also provided great utility in pedestrian wayfinding as they also possess the same level of permanence as their intersection navigation counterparts used in vehicle wayfinding [4].

The physiological factors impacting route choice for pedestrian navigation are important as well. As mentioned above, mental satisfaction [1] is a prime driver as to why pedestrians choose a route which may not be the shortest route. Other motivators such as safety, convenience, and 
attractiveness of a route have been shown [7] to strongly influence route choices. The findings from [7] demonstrate that while people believe time and distance are their primary or sole factors for route selection, other factors do have a strong influence, and direct and safe routes are among the top criteria. [8] recommends a fuzzy logic that could consider such options. The logic implemented allows for each sidewalk segment to be weighted according to pedestrian preferences, or know information about pedestrians such as in [7], so when determining routes, factors outside of path length can be considered.

\section{Standards: ADAAG}

Resources such as the ADAAG, the Architectural Barriers Act (ABA) [3], and the pedestrian access guidelines set forth by the Federal Highway Administration (FHWA) all provide good references for laws and guidelines that have been put into place for PWDs. The ABA, enacted in 1968, for example has clear stipulations for the parameters of entrance doors, entrance vestibules, and ramps that provide access to buildings that are owned by or leased from the federal government, or altered or leased with certain federal grants and loans [9]. The ADAAG, enacted in 1990, and guidelines set forth by the FHWA also state parameters for the width, texture, slope, curb ramps, and obstructions for sidewalks and other pedestrian paths [3]. These laws and guidelines provide clear evidence that there are unique navigational challenges for PWDs and suggest that even people without recognized disabilities may still have requirements or preferences that are unique from other people and are certainly unique from vehicle wayfinding.

\section{Wayfinding for PWDs}

Wayfinding technology for PWDs and the elderly has been a continually progressing field. Studies have recently been conducted that closely analyze the mobility issues pedestrians encounter in everyday situations [10]. Through [10] and others it has been noted that this is not a simple problem as the routing needs of mobility impaired individuals vary depending on the nature of their impairment [11-13] requiring consideration of many variables for solutions. Variables such as sidewalk width, surface, smoothness, and slope are all important considerations, let alone if sidewalks even exist that cover the route being considered from start to finish [12]. In addition, the quality of the sidewalks and pathways need to be considered as well. Narrow sidewalks, poor path surfaces, lack of ramps or exceptionally steep ramps, lack of or blocked curb cuts, sidewalk obstructions, and bad weather are important concerns for mobility impaired individuals as well [14]. Given the multiple variables that need to be factored into creating truly accessible pathways for mobility impaired pedestrians, it can be observed that accessibility is hard to define and is not a well-defined problem [13]. It is also important to note that accessibility varies from person to person regardless of their level of mobility impairment, as each person has different needs for accessibility [11-13].

Different approaches have been proposed to address these concerns. Among them, approaches for personalized routing and navigation have produced positive results. [14] created an algorithm that utilizes fuzzy logic to personalize routes based on user input. The algorithm, taking a fuzzy logic approach to account for individual needs and preferences, rated paths for wheelchair navigation from 0 to 5 , with 5 being most difficult and 0 being impassible. The study found that although personalized routes took longer to travel, they were preferred by wheelchair users for their ease of travel. It is also noteworthy that path surface was the feature wheelchair users mentioned as the primary factor in wayfinding most often. [12] followed up on the work of [14] by further including a reliability factor score to introduce a novel approach to creating requirement-based custom paths and providing an evaluation of the given routes.

Another approach is crowdsourcing which allows volunteers to contribute information to services, such as Open Street Maps (OSM) [15], that are important to pedestrian navigation and are currently not available [11]. An issue with crowdsourced data lies in the reliability of the data [11]. To address this issue, tools have been created to assess the quality of data in mapping environments. Examples of crowdsourcing services are CAP4Access [16], OSMatrix [17], AXS Map [18], Project Sidewalk [19], RouteCheckr [20], and Campus Partner [21]. Through crowdsourcing services volunteers can submit navigation information such as the variables we mentioned above (e.g., path surface conditions, obstructions, and obstacles). The volunteer-provided data in conjunction with the reliability auditing capability of crowdsourcing services help fill in the missing information needed in such services so that personalized navigation for people of varying mobility levels can be realized.

Personalized routing through crowdsourcing is valuable and provides the desired routing solutions for a variety of mobility needs. The IMAGINE (Interactive, Mobile, AGIle, and Novel Education) service [22] has conceptualized this combination and was designed for a community like students in a university campus where it could assist students with disabilities to find and access various campus resources. IMAGINE supports wayfinding options, both algorithmically and socially. Routes in IMAGINE are based on the ADAAG for travel on sidewalks by wheelchair users. IMAGINE was tested with a group of wheelchair participants filling out a questionnaire before and after they tested it. Results found that IMAGINE could improve the participants participation in their community, raise their awareness of wayfinding/navigational 
difficulties in their community, increase their desire for more accessibility in their surroundings, and heighten their awareness of restrictions/boundaries placed on their mobility. However, IMAGINE, as a proof of concept, is a research tool and currently is not available in open platforms.

\section{Wayfinding applications}

A plethora of wayfinding applications are available online and via mobile applications. Google Maps, MapQuest, and Bing Maps are but a few options that provide walking wayfinding options. However, pedestrian wayfinding is not what they are optimized for, as shown in Fig. 1. They do not include pathways that take ADAAG, ABA, or other FHWA standards into account in order to address the mobility needs of PWDs. Regular and recent criticisms such as "Why Google Maps and Citymapper are terrible for walking directions" can be found that note these shortcomings [23]. Some commercial applications do exist that focus on pedestrians, but they all focus on tracking or route planning as opposed to wayfinding. As of this writing, SideKix is the only commercial pedestrian wayfinding application which has a primary focus in points of interest but does not consider personalized wayfinding. Nor do any of the above listed applications provide wayfinding by landmarks. Also, while researched-based applications do exist for either pedestrian wayfinding with landmark instructions or accessible pathways, the authors are unaware of any application, commercial or research, that provides both options.

New services and tools, such as OSM and CityGML, that can be used for pedestrian wayfinding applications are increasingly emerging. Such services can store appropriate data and information, such as landmarks, that can be used for pedestrian wayfinding. However, since none of the current services support accessibility data compliant to the ADAAG, ABA, or FHWA standards, they are unable to provide personalized wayfinding solutions for PWDs.

A recent tool called Urbano, a simulation environment for professional architects, city planners, and research groups, is worth mentioning. As of November 2019, Urbano has been downloaded over 400 times by professional architectural firms and universities [12]. Its primary function is to simulate foot traffic patterns given the proximity of amenities such as schools, libraries, banks, restaurants, and cafes to people. The simulation setup and execution are performed in a three-step process [25]. The simulation environment is created by loading information from resources such as OSM, Google, Yelp, and the New York City Open Data Initiative into the software. The simulations executed by this software produce three metrics [25]. The first metric is a count of how many people used a given street segment during the simulation. This is known as the street score. The second metric is a score from 0 to 100 that rates the ability of people to walk to an amenity based on people's distance from the amenity during the simulation. This is known as the walkability score. The third score is a measure of the difference between the demand for a given amenity and the supply the amenity can produce. This score is derived in the simulation by the number of people that enter the amenity compared to the capacity of the given amenity. This is known as the amenity score. Together these scores can be used to solve realworld problems quickly and cheaply by analyzing existing and proposed models in simulation environments. Several examples are included in [25]. In one example, two neighborhoods were compared to see which had better proximity to amenities overall. In another example, route segments were compared to see which were traveled by people with interests in different amenities. In their final example, individual simulations were run for each of the proposed locations for a new amenity to determine the best location.

The goal of the Urbano software is to promote mobility-aware urban design [25]. The Urbano software has accomplished this if one measures mobility by the distance and convenience of amenities to people. However, other factors such as personal safety and weather protection were specifically chosen not to be included because of the developers' belief in the introduction of bias into the system as a result [25]. This is a gap and opportunity for accessible pathways data in simulation environments. Features outside of route distance play a large factor in foot traffic patterns [1] and features outside of route distance may make some paths completely unusable for people of different mobility needs [14]. These factors would likely impact results produced by simulations in a significant way as often pedestrian prefer a pathway that is not the shortest. There are other opportunities outside of simulation accuracy that can be realized with the inclusion of accessibility data as well. A new series of questions can be answered by the simulation software with the inclusion of accessible pathways data. Is an amenity being underutilized because there are mobility challenges in wayfinding to the amenity that causes a signification portion of people to choose other routes and amenities? Are there entire sections of a neighborhood that are being avoided because mobility, safety, or weather concerns make them challenging? These are valuable questions for both architects and city planners.

Similar to our work, an ADE for CityGML was developed called the Inclusive Routing ADE [24], to provide data that is useful for PWDs. We believe that while, in principal, our work and this Inclusive Routing ADE meet the same objectives and address the same target 
population, our work is different in two aspects. First, in our work we provide the complete process from what PWDs need (include data that meets PWDs needs and preferences for navigating the built environment with comfort), what application developers need (help them develop navigation applications that are useful to PWDs), and how the new ADE can be used by PWDs (help all stakeholders, e.g., PWDs and developers, get a feel for how this new ADE can be used for real-world scenarios using real data). Second, the types of data we consider in our work are based on well-established ADAAG which have been used to address a variety of challenges faced by PWDs over the years.

\section{Ontology for wayfinding of PWDs}

We end this background section by bringing the attention of the researchers and practitioners working on solutions of wayfinding for PWDs to wayfinding ontologies. As an ontology plays an important role in conceptualizing a thing, in our case wayfinding, understood and agreed by all stakeholders, in our case people with disabilities and developer of wayfinding applications, among others, we present in Fig. 3 an ontology [26] for the wayfinding needs and preferences of PWDs highlighting the key elements and the relationships among them. This ontology was specifically designed for the needs and preferences of people with disabilities and was used as the foundation for the work in this paper. The ontology is focused on outdoor wayfinding with important elements, such as obstacles and barriers pertaining to people using wheelchairs, and highlights the types of elements delineated from the ADAAG .

\section{CityGML and ADE-AP}

CityGML is an open source environment used for the storage and retrieval of virtual 3D city models [2]. It stores information for the model in XML files. The CityGML model sits on top of the GML model which uses XML to define geographic objects such as points, lines, areas, and volumes. The CityGML model is able to reference the GML model objects to construct city objects such as buildings, bridges, city furniture, and roads from the shapes stored in the GML model [27]. The CityGML model is also able to store attributes about the objects such as color, material, and texture. The CityGML model allows for easy sharing of data by

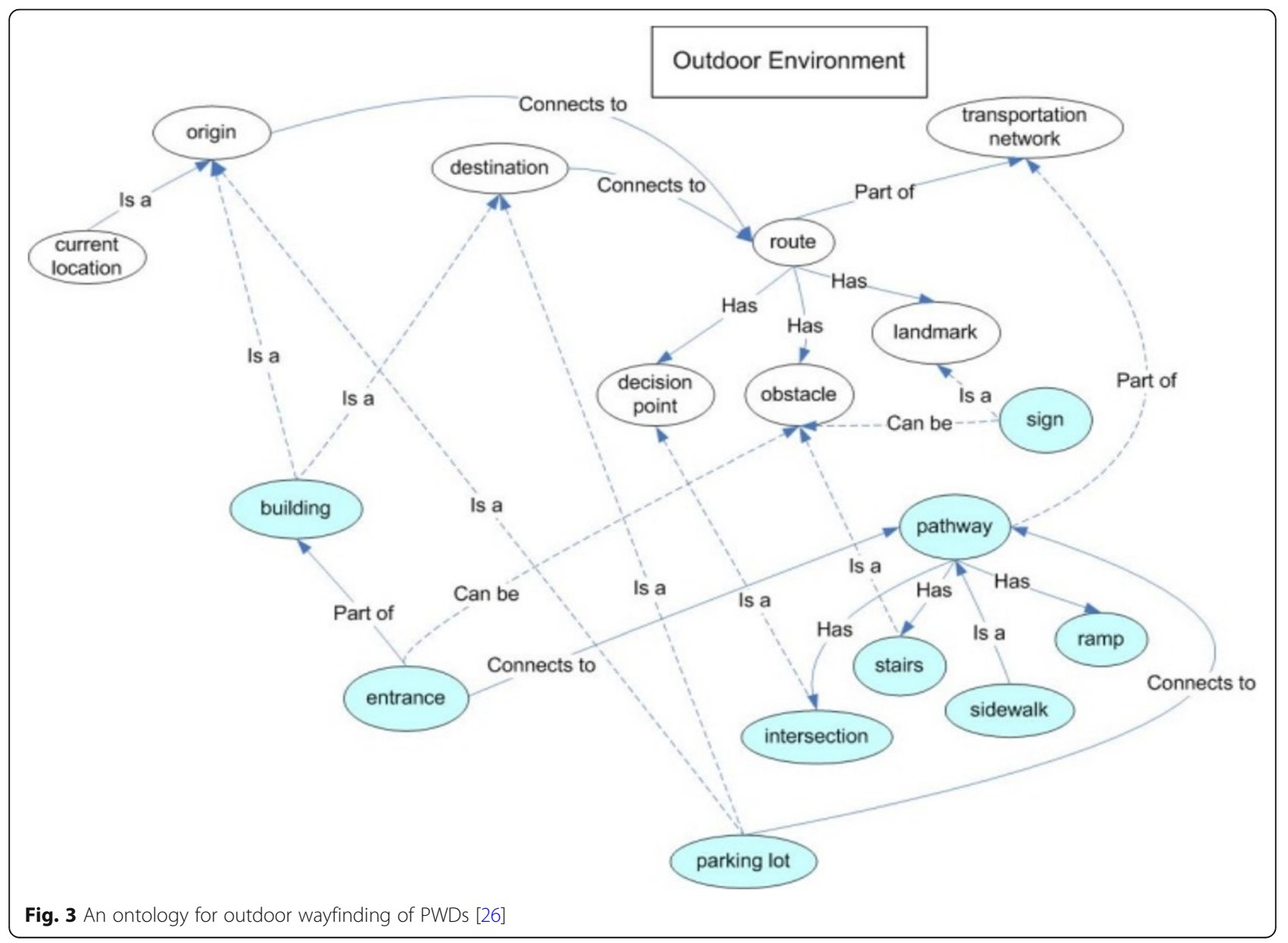


utilizing XSD files. XSD is used to describe the objects within an XML file. CityGML is based on a defined set of XSD files that are maintained by the CityGML community and are available for download from its website [28]. The XSD standards created by the CityGML community comply with six different widely utilized open formats including the ISO 191XX family, the Open Geospatial Consortium, the W3C Consortium, the 3D Consortium, and OASIS (the Organization for Advancement of Structured Information Standards) [27]. The compliance to these standards and the utilization of a central XSD format allow users (developers) utilizing this format to easily share models with each other. Collaborators can pass their XML models back and forth between each other and use the XSD standards to parse the XML file into their desired format. Parsers for XML files via XSD are readily available for most languages, and for our work we use "citygml4j" to parse the files in java [29].

CityGML is both a model and an encoding. It models and encodes city objects allowing to perform operations such as analysis, visualization, and simulation on the data collected. This is possible due to the hierarchical relationships of city objects that are architected into the modeling and encoding [27]. CityGML encodes relationships (i.e., parent-child, sibling-sibling) in CityGML models through object-oriented principles. These encodings can be seen in the specification [27] through their use of UML arrows which document the inheritance, aggregation, association, dependency, and composition between objects. These encodings allow us to directly relate objects such as accessible paths and bus stops, as the former is a type of transportation object and the latter is a city furniture object, both of which inherit from the city object as their base class.

Since CityGML allows modeling of so many different structures within a city, it has become a widely used tool by researchers and practitioners, and has been applied to many domains [30]. It has been used in urban planning, such as building energy simulations [31] and wind simulations [32], in disaster management for flood [33] and earthquake simulations [34], and in time series analysis for sustainable lining, among other things. Because CityGML allows the storage and retrieval of 3D data, its use has also been explored for building virtual and augmented reality for navigation applications.

The features of CityGML make it an excellent choice in our work for several reasons. First, as mentioned in the introduction section, the CityGML model is extendible through its ADE protocol. This is particularly important since CityGML lacks data content for wayfinding of PWDs allowing them to find accessible pathways. Second, CityGML models are highly portable across software and environments as they are based on open standard XML and XSD formats. Third, CityGML, being a free and open platform, will make our proposed ADE-AP available through XML and XSD files to broad range of users and developers.

\section{Proposed ADE-AP}

The existing CityGML model has no defined space for storing accessible pathway data. This is the gap being filled in by our proposed ADE-AP which can be used to develop wayfinding applications for PWDs. We evaluated two options in CityGML to fill this gap. One is to create generic objects within the CityGML model, another is to develop our own ADE-AP. An advantage to creating generic objects is that it can be quickly completed within the existing model with no additional definitions required. However, this may become an issue for collaboration since the generic objects may not be easily used by potential collaborators without some definition in the future. By contrast, developing a new ADE-AP does require time and effort up front to define our new model and relate them to the existing model. We decided on the ADE-AP option since it offers an instant level of robustness and usability that is not afforded by the generic object model.

\section{Accessible pathways}

Our proposed ADE-AP and how it is fit ("Accessible Pathways") within CityGML is shown in Fig. 4. The choice of including this ADE-AP on top of everything else in CityGML allows us to easily add accessible pathway attributes to the existing transportation pathways that exist in the CityGML model. This also allows us to easily relate city furniture to our accessible pathways through the transportation and CityGML layers. In this way we can query the model to obtain both accessible pathway data and city furniture and develop personalized accessible pathways for different pedestrian needs and preferences, in particular those with disabilities, that include landmarks.

The accessible pathway features we have chosen to include in our ADE-AP are discussed in detail in [14, 22], which are focused on addressing the wayfinding of wheelchair users. These ADAAG compliant features for wheelchair users traveling on sidewalks would also provide pathways for the majority of pedestrian wayfinding. Under the ADAAG, each sidewalk segment must comply with the following standards to be considered feasible for traveling by PWDs [3]:

- Width should be $92 \mathrm{~cm}$ wide with no obstructions.

- Slope should not exceed 5\% and cross slope should not exceed $2 \%$.

- Should have a firm, stable, and slip resistant walking surface.

- Should have a concrete, asphalt, stone, or brick surface. 
- Should be void of major cracks or breakage. Should support curb ramps where applicable.

It must be noted that having these ADAAG in ADE-AP is part of the solution and the other part is developing algorithms that can utilize these ADAAG appropriately and in a way that personalized accessible pathways can be computed.

The transportation thematic model in CityGML allows the storage or derivation of information for sidewalk segment width, length, grade, and cross slope. The city furniture thematic model in CityGML contains information about the locations of ramps and steps. In addition, surface condition and surface type according to the ADAAG are also included in ADE-AP. Currently, through this work presented in this paper, while our proposed ADE-AP only supports these data mentioned above, which are suitable for wheelchair users, it provides the foundation for adding other data such as sidewalk foot traffic, crime rate, personal safety, lighting, and weather condition. Figure 5 shows the details of how the proposed ADE-AP is included in CityGML. ADE-AP extends th "Track" feature of CityGML which includes the general features for pedestrians [27]. We have set the level of detail (LOD) for all our variables in the accessible pathways to 3 . The accessible pathway features are represented as a MultiSurface in LOD 2 and subtle objects such as manholes or roadway damages are represented as LOD 3 [35]. Also, in LOD 3, the accessible pathway will have a linear representation.

\section{ADE-AP in CityGML}

CityGML creates and relates city features in an objectoriented fashion. Figure 5 shows that accessible pathways derived from ADE-AP are associated with instances of track features. This allows us to provide accessible pathway data for each track instance. Each track instance inherits from the transportation complex feature, which inherits from the transportation object feature, which inherits from the city object feature. The city object feature is the base object from which all CityGML thematic models inherit. Therefore, accessible pathways through ADE-AP can access city furniture thematic models as well and can include landmarks in wayfinding.

Given the object-oriented nature of CityGML, the creation of models that can be used in personalized wayfinding can take on several forms. In the simplest form, if one only care about accessible pathways, an entire model based on track features alone can be generated. If one wishes to add landmarks in wayfinding solutions, the inclusion of city furniture features will be required as well. As mentioned above, since these all come from the same base class, the track features can see the city furniture features and can therefore seamlessly create personalized landmark-based wayfinding solutions. This model can further be expanded if a detailed model of the city is developed.

\section{Experiments}

We conducted experiments to demonstrate the operational workings of our proposed ADE-AP. In these experiments we used the sidewalk data that are compliant to the ADAAG and the algorithms that were designed for the specific mobility needs and preferences of people relying on wheelchairs for daily mobility activities. We generated ADE-AP-driven accessible pathways and compared them with the pedestrian pathways generated by Google Maps. Note that in these experiments in this work, Google Maps, for its global availability and popularity, is chosen as a representative wayfinding solution commonly used by individuals, including those with disabilities.

\section{Accessible Pathways

\begin{tabular}{l|l|l|l} 
Transportation & City Furniture & Buildings & etc...
\end{tabular}

CityGML

GML

XML

Fig. 4 Our proposed ADE-AP ("Accessible Pathways") and its place in CityGML 


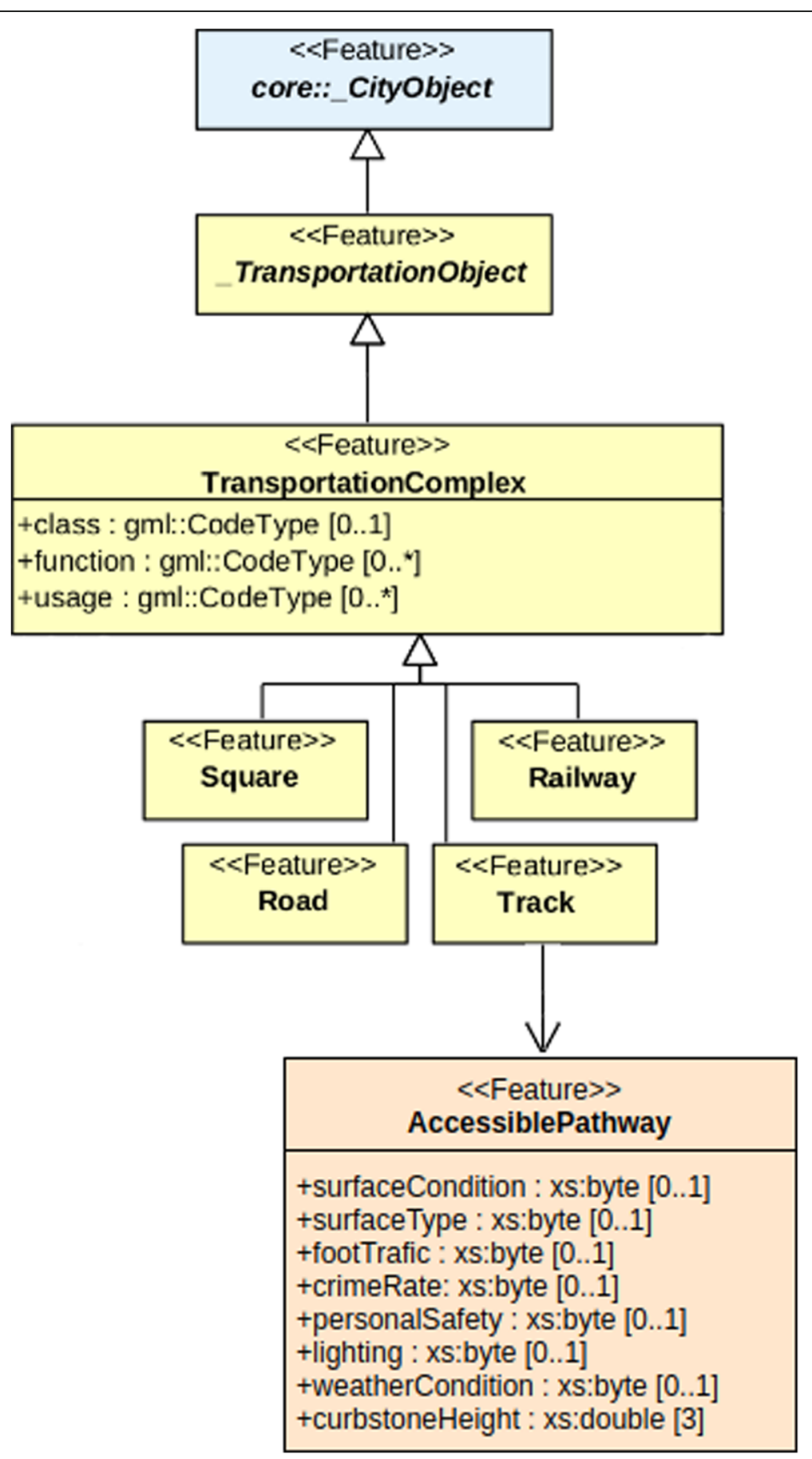

Fig. 5 ADE-AP details

\section{Data}

We used the sidewalk network data within the University of Pittsburgh's main campus in the experiments (Fig. 6) [36]. The data was collected manually by one of the researchers in the Geoinformatics Laboratory of the School of Computing and Information at the University of Pittsburgh and by taking into account the ADAAG. Various devices and tools, such as GPS and tape measures, were used in collecting width, length, slope, surface type, surface condition, traffic, and steps of each sidewalk segment.

We incorporated the collected sidewalk data in the CityGML model to develop ADE-AP. We used the citygml4j library [29] of the java programming language and tested the sharing of the new data with others by a private network shared drive. One team member 


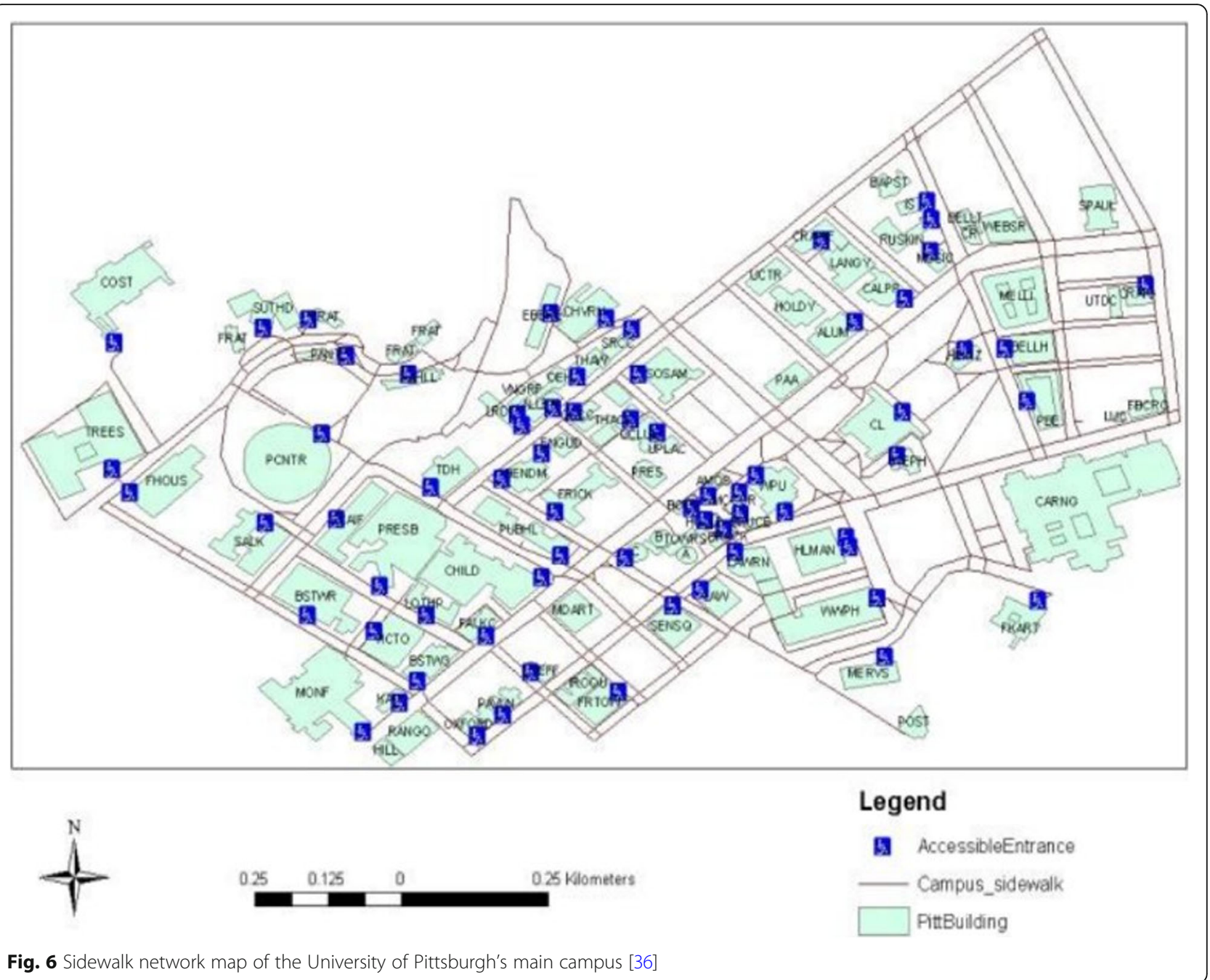

uploaded the model and XSD file from a computer to the drive, another downloaded the model and XSD file to another computer. Then the same citygml4j library was utilized to parse the model and load it into ArcGIS as shapefiles to generate accessible pathways. We developed our own accessible pathway XSD so the library could correctly handle the parsing of the data into our new accessible pathway model.

\section{Accessible pathways generation}

In the experiments, we generated routes (pathways) by three different approaches: ADE-AP approach, PAM (Personalized Accessible Mapping) approach, and Google Maps approach. PAM [37] was developed by researchers in the Geoinformatics Laboratory of the School of Computing and Information at the University of Pittsburgh. In both ADE-AP and PAM approaches, we utilized the sidewalk network data of the University of Pittsburgh's main campus and a fuzzy logic algorithm [14] to take into account the seven ADAAG-compliant parameters (width, length, slope, surface type, surface condition, traffic, steps) to reflect an individual wheelchair user's weight for each sidewalk segment. The fuzzy logic algorithm, by utilizing the provided weight on each parameter for each sidewalk segment, calculated an impedance score between 0 and 5 , with 5 being the most difficult to travel and 0 being impassible. The impedance score factors in the user defined needs and preferences in addition to the physical characteristics of the sidewalk segments, all based on the ADAAG. Once the impedance score for an individual was calculated, it was assigned as weight for the segment, which in turn was used by Dijkstra's algorithm to find personalized route on the sidewalk network of the campus.

It should be noted that the pathways produced by our ADE have already been tested by PWDs for addressing their needs [14]. We used the pathways from Google Maps to compare with the pathways produced by our ADE for three reasons. One reason is the fact that Google Maps is used worldwide and has become a de facto tool for navigation 
(driving, walking, etc.) without knowing the quality pathways it produces. Another reason is that we wanted to demonstrate the gap in a current commercial widespread tool in addressing mobility challenges of PWDs. Our third reason is to show that without using widely agreed standards (like ADAAG), navigation options provided by current tools are not feasible, safe, or comfortable for PWDs.

We generated accessible pathways of varying lengths and compared them to pedestrian paths generated by Google Maps. We generated five accessible pathways: long (greater than $1.29 \mathrm{~km}$ ), medium (between 0.64 and $1.29 \mathrm{~km}$ ), and short (less than $0.64 \mathrm{~km}$ ) distances for walking directions around the University of Pittsburgh's main campus. Since there are no reasons for taking the same pathways when travelling between two locations (origin to destination and destination to origin), we generated return routes for each of the pathways we generated. This provided us a total of 120 routes.

To generate accessible pathways through the ADE-AP approach, we retrieved sidewalk data from ADE-AP in CityGML, calculated impedance score for each sidewalk segment by applying the fuzzy logic algorithm and using different values for the seven parameters, stored the sidewalk network data along with the impedance score for each sidewalk segment in ArcGIS and ran the routing algorithm (Dijkstra's algorithm). The reason we used the
ArcGIS Network Analyst extension is that it is a widely utilized tool by many organizations and communities around the world for performing some transportation computation and analysis, such as finding the shortest path. We also generated accessible pathways in PAM and used them as ground truths for comparisons. The reason for using accessible pathways in PAM, which uses the same sidewalk network data of the University of Pittsburgh's main campus, the same fuzzy logic to calculate impedance scores for sidewalk segments, and Dijkstra's algorithm for finding pathways, as ground truths is that accessible pathways in PAM were designed for and tested by wheelchair users [37]. As for pedestrian pathways generated through the Google Maps approach, due to the proprietary nature of commercial services, we do not know the details of the data it uses nor the algorithms for computing routes.

For each generated pathway, we measured length, number of turns, and number of segments as metrics to compare the different pathways (accessible pathways through ADE-AP and PAM and pedestrian pathways through Google Maps). The pathway length was the sum of all the pathway segment lengths. The number of turns included any change of direction onto a new street greater than 60 degrees as shown in Fig. 7. Number of

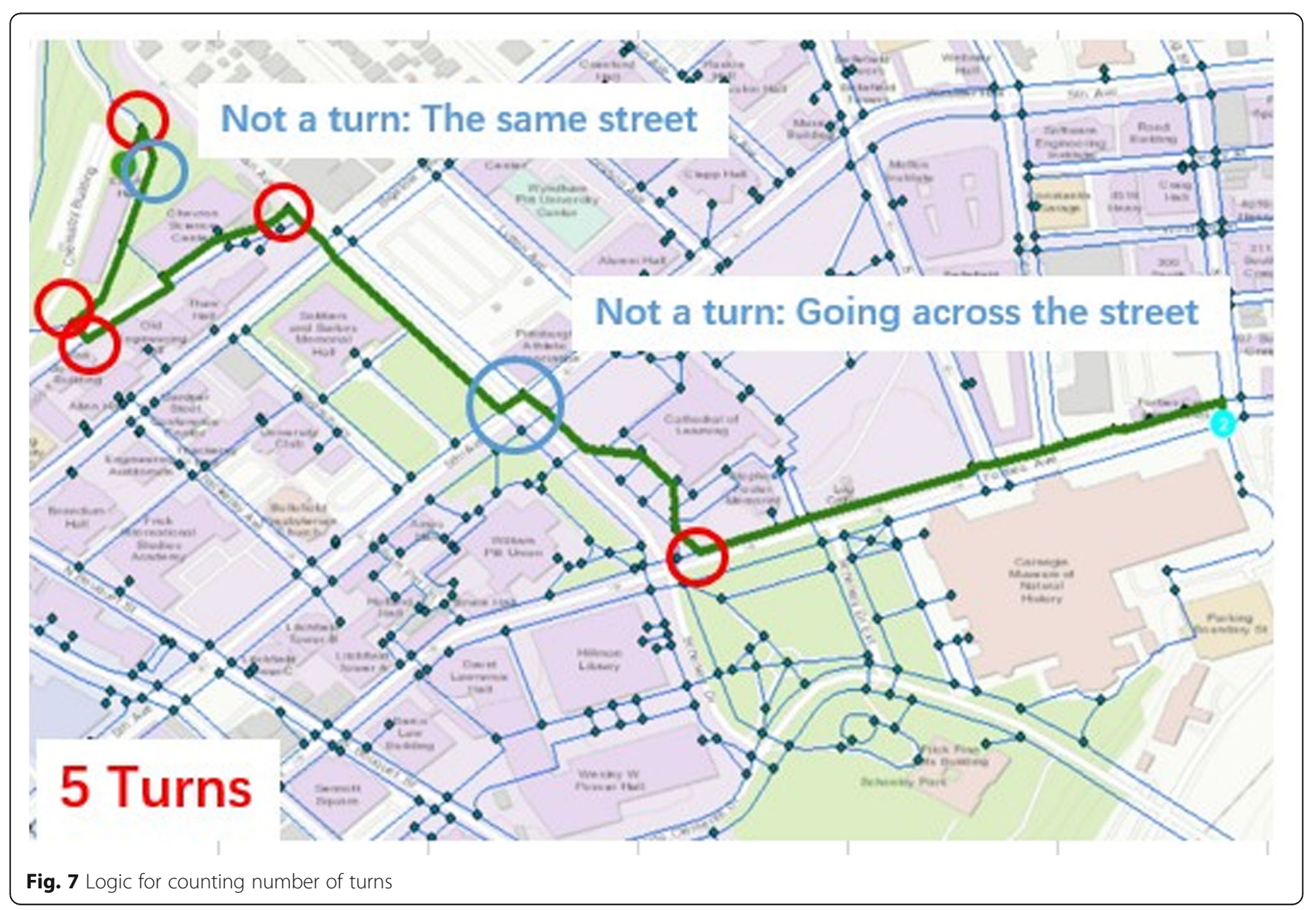


segments provides a count of the route segments that exist in an generated accessible pathways generated by ADE-AP and PAM. However, since we did not have access to the path segments data and algorithms used in generating pedestrian pathways by Google Maps, we used the number of instructions in the pedestrian pathways generated by Google Maps in its place.

\section{Results and discussion}

Figure 8 shows an example pathway generated by ADEAP, PAM, and Google Maps. The pathways in this figure are of a medium length of approximately $0.64 \mathrm{~km}$ and the accessible pathways are shown in green (ADE-AP) and in blue (PAM) and the pedestrian (shortest pathways) in dotted blue (Google Maps). Table 1 shows the results of the metrics for all pathways generated through the three different approaches. In can be seen in this table that all pathways produced similar lengths and number of turns on average, but the number of segments produced by each approach varies greatly.

It is important to note that the pathways produced by our ADE are all feasible based on ADAAG. We decided those three metrics (i.e., length, number of turns, and number of segments) to evaluate our ADE solutions based on the observation that once it is determined that a pathway is passible, PWDs are interested in checking to see whether the pathway meets their preferences and comfort levels either directly or indirectly. We believe that these metrics are representative of these preferences and comfort levels.

Table 2 shows the result of the Mann-Whitney $U$ test applied to the metrics to determine the similarities

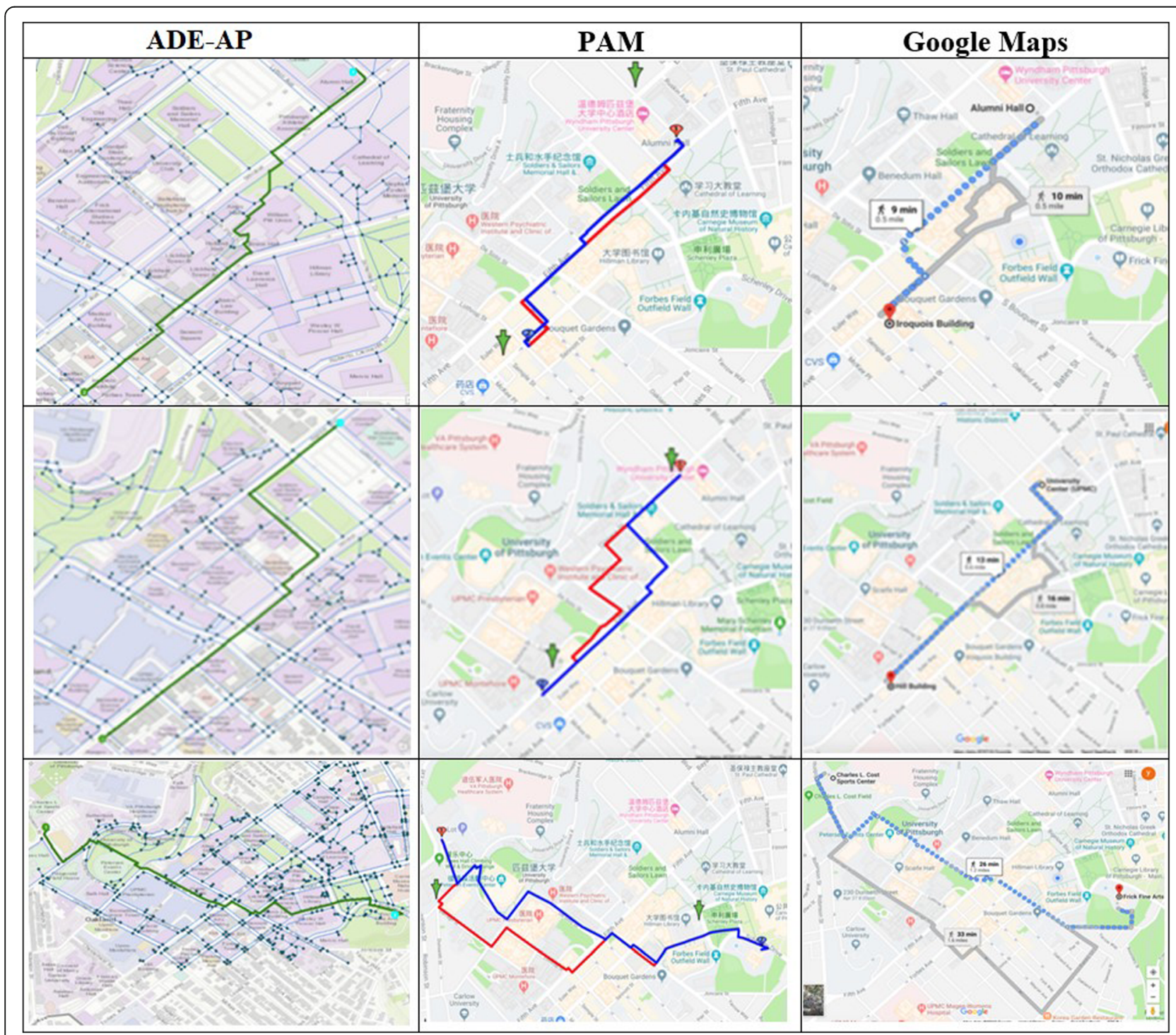

Fig. 8 Paths generated through ADE-AP, PAM, and Google Maps 
Table 1 The average pathway length, number of segments, and number of turns for each approach for all routes generated

\begin{tabular}{llll}
\hline System & Length $(\mathrm{km})$ & Segments & Turns \\
\hline ADE-AP & 0.92 & 8.09 & 4.03 \\
PAM shortest & 0.98 & 14.6 & 3.6 \\
PAM accessible & 0.98 & 15.87 & 3.2 \\
Google & 0.92 & 6.23 & 3.73 \\
\hline
\end{tabular}

between the routes. The null hypothesis for the test is to check the likelihood of a randomly selected value from one population being less than or greater than a randomly selected value from a second population. Therefore, a p-value of 0.5 shows equivalence. As we can see from Table 2, there is no strong equivalence among our metrics between the three different approaches.

The results of our above experiments highlight our main contribution of the paper, i.e., storing ADAAG compliant data in a free and open platform (i.e., CityGML) and retrieving and utilizing it in applications and tools (in this case ArcGIS software) to find accessible pathways that meet the mobility requirements of PWDs.

By comparing the accessible pathways obtained through ADE-AP with the pedestrian pathways obtained through Google Maps the following are observed: (a) commercial tools/services by private companies (such as Google) do not provide complete accessible pathway solutions for PWDs; (b) even when/if commercial tools/services provide complete accessible pathway solutions, they may not be compliant to standards such as the ADAAG; (c) commercial tools/services supported by private companies, due to the proprietary nature of their business, do not allow access to their resources by others to be used in wayfinding applications; and (d) our proposed ADE-AP paves the way for the inclusion of additional data for all people with all different types of disabilities in CityGML.

\section{Conclusion and future research directions}

In this paper, we highlighted the gap in current wayfinding applications and services where they are not able to meet the wayfinding requirements of PWDs. In doing so, we discussed two main important elements. One is that the

Table 2 Mann-Whitney $U$ test to compare distributions of pathway lengths, number of segments, and number of turns between two approaches for all routes generated by each approach

\begin{tabular}{lllll}
\hline System1 & System2 & Length & Segments & Turns \\
\hline ADE-AP & PAM shortest & 0.284 & 0.00000212 & 0.1954 \\
ADE-AP & PAM accessible & 0.289 & 0.0005 & 0.2929 \\
ADE-AP & Google & 0.4615 & 0.0444 & 0.4261 \\
PAM accessible & Google & 0.2995 & 0.00000288 & 0.1746 \\
Google & Google & 0.2842 & 0.000000023 & 0.0785 \\
\hline
\end{tabular}

accessibility data used for finding accessible pathways for PWDs must be compliant to widely agreed upon and available standards. Another is that the accessible data must be made available in free and open platforms so that it can be used by developers to develop personalized wayfinding applications and services. We discussed our contributions, providing accessibility data based on the ADAAG for PWDs and developing a new ADE (ADE-AP) in CityGML to facilitate the development of wayfinding applications and services for PWDs to fill this gap. Through experiments, we demonstrated how we could build, share, and use the new ADE-AP in CityGML and generate accessible pathways which we compared with pedestrian pathways generated by Google Maps.

In this work, we focused on accessibility data and accessible pathways for wheelchair users, but our proposed ADE-AP allows inclusion of accessibility data that meet other mobility challenges, such as people who are blind or are visually impaired. Future research directions include development of simulations in smart cities for analyzing accessible pathways for all different individuals, mobility abled and mobility impaired, and development of virtual reality and augmented reality for mobility in smart cities.

\section{Acknowledgements \\ Not applicable.}

\section{Authors' contributions}

All authors jointly developed the project idea and performed research. BW created a model and architecture. YW and RG tested the model. All authors read and approved the final manuscript.

\section{Funding}

No funding provided for this article.

\section{Availability of data and materials}

The datasets are available from the corresponding author on reasonable request.

\section{Competing interests}

The authors declare that they have no competing interests.

\section{Author details}

1 Geoinformatics Laboratory, School of Computing and Information, University of Pittsburgh, 135 N Bellefield Ave, Pittsburgh, PA 15213, USA. ${ }^{2}$ School of Computing and Information, University of Pittsburgh, $135 \mathrm{~N}$ Bellefield Ave, Pittsburgh, PA 15213, USA.

Received: 14 January 2020 Accepted: 20 May 2020

Published online: 16 June 2020

\section{References}

1. Fang Z, Li Q, Shaw SL. What about people in pedestrian navigation? Geo Spatial Inf Sci; 2015. https://doi.org/10.1080/10095020.2015.1126071.

2. Gröger G, Plümer L. CityGML - Interoperable semantic 3D city models. ISPRS J Photogrammetry Remote Sensing. 2012. https://doi.org/10.1016/j.isprsjprs. 2012.04.004

3. United States Access Board, Americans with disabilities act and architectural barriers act accessibility guidelines. 2004.

4. Basiri A, Amirian P, Winstanley A, Marsh S, Moore T, Gales G. Seamless pedestrian positioning and navigation using landmarks: J Navig; 2016. https://doi.org/10.1017/S0373463315000442. 
5. Fang Z, Li Q, Zhang X, Shaw SL. A GIS data model for landmark-based pedestrian navigation: Int J Geogr Inf Sci; 2012. https://doi.org/10.1080/ 13658816.2011.615749

6. Rehrl K, Häusler E, Leitinger S. Comparing the effectiveness of GPSenhanced voice guidance for pedestrians with metric- and landmarkbased instruction sets. In: Lecture notes in computer science (including subseries lecture notes in artificial intelligence and lecture notes in bioinformatics); 2010. https://doi.org/10.1007/978-3-642-15300-6_14.

7. Agrawal AW, Schlossberg M, Irvin K. How far, by which route and why? A spatial analysis of pedestrian preference: J. Urban Des; 2008. https://doi.org/ 10.1080/13574800701804074

8. Ren M, Karimi HA. A fuzzy logic map matching for wheelchair navigation: GPS Solut; 2012. https://doi.org/10.1007/s10291-011-0229-5.

9. U.S General Services Administration, "National Accessibility Program Standards, Policies." [Online]. Available: https://www.gsa.gov/cdnstatic/ National_Accessibility_Program_Standards_Policies_and_Procedures_2.pdf.

10. Tournier I, Dommes A, Cavallo V. Review of safety and mobility issues among older pedestrians: Accid Anal Prev; 2016. https://doi.org/10.1016/j. aap.2016.02.031

11. L. Criscuolo et al., Handing quality in crowdsourced geographic information. 2016

12. Neis $P$. Measuring the reliability of wheelchair user route planning based on volunteered geographic information. Trans GIS. 2015. https://doi.org/10. 1111/tgis.12087.

13. Rahaman MS, Mei Y, Hamilton M, Salim FD. CAPRA: A contour-based accessible path routing algorithm. Inf Sci (Ny). 2017. https://doi.org/10.1016/ j.ins.2016.12.041.

14. Kasemsuppakorn P, Karimi HA, Ding D, Ojeda MA. Understanding route choices for wheelchair navigation: Disabil Rehabil Assist Technol; 2015. https://doi.org/10.3109/17483107.2014.898160.

15. Weber P, Haklay M. OpenStreetMap: user-generated street maps: IEEE Pervasive Comput; 2008.

16. "CAP4Access." [Online]. Available: http://www.cap4access.eu/index.php?id= intro.

17. "OSMatrix." [Online]. Available: https://wiki.openstreetmap.org/wiki/OSMatrix.

18. "AXS Map." [Online]. Available: https://www.axsmap.com/.

19. "Project Sidewalk." [Online]. Available: https://sidewalk-sea.cs.washington.edu/.

20. Völkel T, Weber G. RouteCheckr: personalized multicriteria routing for mobility impaired pedestrians. In: ASSETS'08: the 10th international ACM SIGACCESS conference on computers and accessibility; 2008. https://doi. org/10.1145/1414471.1414506.

21. Zastudil C, Stewart M, Sprague N, Brady E. Campuspartner: an assistive technology for mobility impaired pedestrians. In: Proceedings of the international ACM SIGGROUP conference on supporting group work; 2020. https://doi.org/10.1145/3323994.3369893.

22. Goldberg M, Karimi H, Pearlman JL. Interactive, mobile, AGlle and novel education (IMAGINE): a conceptual framework to support students with mobility challenges in higher education. Disabil Rehabil Assist Technol. 2016;11(1):50-60.

23. N. Kobie, "Why Google Maps and Citymapper are terrible for walking directions." [Online]. Available: https://www.wired.co.uk/article/walkingdirections-air-pollution-google-maps.

24. Prandi F, et al. Using CITYGML to deploy SMART-city services for URBAN ecosystems. ISPRS Int Arch Photogramm Remote Sens Spat Inf Sci. 2013. https://doi.org/10.5194/isprsarchives-xl-4-w1-87-2013.

25. Dogan T, Samaranayake S, Saraf N. Urbano: A new tool to promote mobility-aware urban design, active transportation modeling and access analysis for amenities and public transport. In: Simulation Series; 2018 https://doi.org/10.22360/simaud.2018.simaud.028.

26. Karimi HA, Hashemi M. Accessible wayfinding testbed: infrastructure and components. In: EAl international conference on Testbeds and research infrastructures for the development of networks and communities (TRIDENTCOM); 2015. https://doi.org/10.4108/icst.tridentcom.2015.259709.

27. G. Gröger, T. Kolbe, C. Nagel, and K.-H. Häfele, "OGC City Geography Markup Language (CityGML) En-coding Standard," Ogc, 2012, doi: OGC 12-019

28. "CityGML." [Online]. Available: https://www.ogc.org/standards/citygml.

29. C. Nagel, "citygm/4j-the open source Java API for CityGML." Version, 2017.

30. Biljecki F, Stoter J, Ledoux H, Zlatanova S, Çöltekin A. Applications of 3D city models: State of the art review. ISPRS Int J Geo Inf. 2015. https://doi.org/10. 3390/ijgi4042842.
31. Nouvel R, et al. Development of the citygml application domain extension energy for urban energy simulation. In: 14th International Conference of IBPSA - Building Simulation 2015, BS 2015, Conference Proceedings; 2015.

32. Saeedrashed YS, Benim AC. Validation methods of geometric 3D-CityGML data for urban wind simulations. In: E3S web of conferences; 2019. https:// doi.org/10.1051/e3sconf/201912810006

33. Singh H, Garg RD. Web 3D GIS application for flood simulation and querying through open source technology. J Indian Soc Remote Sens. 2016 https://doi.org/10.1007/s12524-015-0498-5.

34. Christodoulou SE, Vamvatsikos D, Georgiou C. A BIM-based framework for forecasting and visualizing seismic damage, cost and time to repair. In: eWork and eBusiness in Architecture, Engineering and Construction Proceedings of the European Conference on Product and Process Modelling 2010; 2010. https://doi.org/10.1201/b10527-8.

35. Beil C, Kolbe TH. Citygml and the streets of new york - a proposal for detailed street space modelling. In: ISPRS annals of the photogrammetry, remote sensing and spatial information sciences; 2017. https://doi.org/10. 5194/isprs-annals-IV-4-W5-9-2017.

36. Kasemsuppakorn $P$, Karimi HA. Data requirements and a spatial database for personalized wheelchair navigation. In: I-CREATe 2008 - international convention on rehabilitation engineering and assistive technology 2008; 2008.

37. Karimi HA, Zhang L, Benner JG. Personalized accessibility map (PAM): a novel assisted wayfinding approach for people with disabilities. Ann GIS. 2014. https://doi.org/10.1080/19475683.2014.904438.

\section{Publisher's Note}

Springer Nature remains neutral with regard to jurisdictional claims in published maps and institutional affiliations.

\section{Submit your manuscript to a SpringerOpen ${ }^{\circ}$ journal and benefit from:}

- Convenient online submission

- Rigorous peer review

- Open access: articles freely available online

- High visibility within the field

- Retaining the copyright to your article

Submit your next manuscript at $>$ springeropen.com 